\title{
THE STRONGLY CONFINED SCHRÖDINGER-POISSON SYSTEM FOR THE TRANSPORT OF ELECTRONS IN A NANOWIRE
}

\author{
NAOUFEL BEN ABDALLAH, FRANCOIS CASTELLA, FANNY FENDT, \\ AND FLORIAN MÉHATS
}

\begin{abstract}
We study the limit of the three-dimensional Schrödinger-Poisson system with a singular perturbation, to model a quantum electron gas that is strongly confined near an axis. For well-prepared data, which are polarized on the ground space of the transversal Hamiltonian, the resulting model is the cubic defocusing nonlinear Schrödinger equation. Our main tool is a refined analysis of the Poisson kernel when acting on strongly confined densities. In that direction, an appropriate scaling of the initial data is required, to avoid divergent integrals when the gas concentrates on the axis.
\end{abstract}

\section{Introduction}

1.1. The physical problem and the singularly perturbed system. Artificially confined structures are now routinely realized in the nanoelectronic industry and the functioning of many electronic devices is based on the transport of charged particles which are bounded in transversal directions, see e.g. [AFS], [Bas], [FG], [VW]. The confinement can be typically monodimensional like in quantum wells where two directions remain for the transport, or bidimensional like in quantum wires where the transport is in dimension one. In this work we are interested in the second case, and this paper is devoted to the rigorous derivation of a dynamic onedimensional quantum model with space-charge effects describing the transport of electrons confined in a nanowire. Our strategy is inspired from the one in [BAMP], $[\mathrm{BMSW}],[\mathrm{BCM}]$ and consists in an asymptotic analysis of the three-dimensional Schrödinger-Poisson system (or Hartree system) -that will be referred to as our "starting model"- with a singular perturbation modeling a strong potential confining the electron gas in a wire. The interesting point concerning the reduced model obtained in the limit is that the nonlinearity describing space-charge effects is now localized, this reduced model taking the form of a cubic defocusing nonlinear Schrödinger equation.

Let us describe the starting model. The space variable is written $\left(x, z_{1}, z_{2}\right)$, where $x \in \mathbb{R}$ is the direction in which the electron gas is transported free from any external force and $z=\left(z_{1}, z_{2}\right) \in \mathbb{R}^{2}$ are the confined directions. We consider the following singularly perturbed Schrödinger-Poisson system:

$$
\begin{gathered}
i \partial_{t} \Psi^{\varepsilon}=-\Delta \Psi^{\varepsilon}+\frac{1}{\varepsilon^{2}} V_{c}\left(\frac{z}{\varepsilon}\right) \Psi^{\varepsilon}+\mathbb{V}^{\varepsilon} \Psi^{\varepsilon}, \\
\mathbb{V}^{\varepsilon}=\frac{1}{4 \pi r} *\left(\left|\Psi^{\varepsilon}\right|^{2}\right) .
\end{gathered}
$$

2000 Mathematics Subject Classification. 35Q40, 35Q55, 35B40, 35B25, 82D37.

Key words and phrases. Schrödinger-Poisson system, asymptotic analysis, singular perturbation, quantum transport, nanowire, nanoelectronics. 
The unknown in this system is the pair $\left(\Psi^{\varepsilon}, \mathbb{V}^{\varepsilon}\right)$ made of the electronic wave function $\Psi^{\varepsilon}$ and the self-consistent potential $\mathbb{V}^{\varepsilon}$ due to space charge effects, written here as a convolution with the Poisson kernel. We use the notation $r=r(x, z)=\sqrt{x^{2}+|z|^{2}}$. The main modeling assumption is that a strong external potential is applied to the gas, written here $\frac{1}{\varepsilon^{2}} V_{c}\left(\frac{z}{\varepsilon}\right)$, where $V_{c}(z)$ is a prescribed function satisfying the following assumption.

Assumption 1.1. The function $V_{c}: \mathbb{R}^{2} \mapsto \mathbb{R}$ belongs to $L_{\text {loc }}^{2}\left(\mathbb{R}^{2}\right)$ and there exists $\alpha>0$ and $C>0$ such that

$$
V_{c}(z) \geq C|z|^{\alpha} \text {. }
$$

The crucial assumption here is the growth at infinity, which determines the strength of the confinement. The parameter $\varepsilon \in(0,1)$ is the scaled thickness of the electron gas. As we will see after a rescaling in the next section, the normalisation term $\frac{1}{\varepsilon^{2}}$ is natural in order to balance the strong external potential with the Laplace operator in the $z$ variable.

This paper studies the asymptotic behaviour of $\left(\Psi^{\varepsilon}, \mathbb{V}^{\varepsilon}\right)$ as $\varepsilon$ goes to zero. Of course, an initial data $\Psi^{\varepsilon}(0, x, z)$ needs to be prescribed for (1.1), whose specific form is made precise in the next section.

1.2. Scaling of the initial data and formal limit. In this section, we derive heuristically the asymptotic model satisfied by the solution of (1.1)-(1.2) as $\varepsilon$ goes to zero. Precise and rigourous statements will be made in the next section. Let us introduce the following notation for averages in the transversal variables:

$$
\langle f\rangle=\int_{\mathbb{R}^{2}} f(z) d z .
$$

The singular term $\frac{1}{\varepsilon^{2}} V_{c}\left(\frac{z}{\varepsilon}\right)$ in the Schrödinger equation (1.1) induces a concentration of the density on the axis $z=0$. We expect that, as $\varepsilon \rightarrow 0$, the density takes the form of a line density multiplied by a delta function:

$$
\left|\Psi^{\varepsilon}(t, x, z)\right|^{2} \sim\left\langle\left|\Psi^{\varepsilon}(t, x, \cdot)\right|^{2}\right\rangle \delta(z) .
$$

The crucial point is the consequence of (1.3) on the selfconsistent potential. Indeed, we can prove (see Proposition 2.1) that, near the axis $z=0$, the solution of (1.2) looks like

$$
\mathbb{V}^{\varepsilon}(t, x, z) \sim-\frac{1}{2 \pi}\left\langle\left|\Psi^{\varepsilon}(t, x, \cdot)\right|^{2}\right\rangle \log \varepsilon
$$

This estimate suggests the following choice of initial data: we choose $\Psi^{\varepsilon}(0, x, z)$ to be small, of order $|\log \varepsilon|^{-1 / 2}$ (e.g. in $L^{2}\left(\mathbb{R}^{3}\right)$ ).

In order to observe the system at the scale of the gas, we work with rescaled space variables, setting

$$
\Psi^{\varepsilon}(t, x, z)=\frac{1}{\varepsilon \sqrt{|\log \varepsilon|}} \psi^{\varepsilon}\left(t, x, \frac{z}{\varepsilon}\right), \quad \mathbb{V}^{\varepsilon}(t, x, z)=\frac{1}{|\log \varepsilon|} V^{\varepsilon}\left(t, x, \frac{z}{\varepsilon}\right) .
$$

The system in the new unknowns $\psi^{\varepsilon}, V^{\varepsilon}$ becomes

$$
\begin{gathered}
i \partial_{t} \psi^{\varepsilon}=-\partial_{x}^{2} \psi^{\varepsilon}+\frac{1}{\varepsilon^{2}} H_{z} \psi^{\varepsilon}+\frac{1}{|\log \varepsilon|} V^{\varepsilon} \psi^{\varepsilon}, \\
V^{\varepsilon}=\frac{1}{4 \pi r^{\varepsilon}} *\left|\psi^{\varepsilon}\right|^{2}, \quad r^{\varepsilon}(x, z)=\sqrt{x^{2}+\varepsilon^{2}|z|^{2}}, \\
\psi^{\varepsilon}(0, x, z)=\psi_{0}^{\varepsilon}(x, z),
\end{gathered}
$$

where the Hamiltonian in the $z$ direction is

$$
H_{z}=-\Delta_{z}+V_{c}(z) \text {. }
$$


Inserting (1.4) in (1.5), we obtain that, asymptotically, $\psi^{\varepsilon}$ satisfies

$$
\begin{gathered}
i \partial_{t} \psi^{\varepsilon}=-\partial_{x}^{2} \psi^{\varepsilon}+\frac{1}{\varepsilon^{2}} H_{z} \psi^{\varepsilon}+\frac{1}{2 \pi}\left\langle\left|\psi^{\varepsilon}\right|^{2}\right\rangle \psi^{\varepsilon}, \\
\psi^{\varepsilon}(0, x, z)=\psi_{0}^{\varepsilon}(x, z) .
\end{gathered}
$$

This is our reduced model. An elementary remark is, the term $\left\langle\left|\psi^{\varepsilon}\right|^{2}\right\rangle$ in the nonlinearity does not depend on the $z$ variable. It is thus easy to filter out the oscillations in time induced by the $\frac{1}{\varepsilon^{2}} H_{z}$ term. Indeed the function

$$
\phi^{\varepsilon}=e^{i t H_{z} / \varepsilon^{2}} \psi^{\varepsilon}
$$

satisfies the following problem, independent of $\varepsilon$ :

$$
\begin{gathered}
i \partial_{t} \phi^{\varepsilon}=-\partial_{x}^{2} \phi^{\varepsilon}+\frac{1}{2 \pi}\left\langle\left|\phi^{\varepsilon}\right|^{2}\right\rangle \phi^{\varepsilon}, \\
\phi^{\varepsilon}(0, x, z)=\psi_{0}^{\varepsilon}(x, z),
\end{gathered}
$$

where we used the fact that $e^{i t H_{z} / \varepsilon^{2}}$ is an isometry on $L_{z}^{2}\left(\mathbb{R}^{2}\right)$, ie

$$
\left\langle\left|\phi^{\varepsilon}\right|^{2}\right\rangle=\left\langle\left|\psi^{\varepsilon}\right|^{2}\right\rangle \text {. }
$$

The limit model can be seen as a system of nonlinear Schrödinger equations (NLS) in dimension one. To see this, let us introduce the eigenfunctions $\left(\chi_{k}(z)\right)_{k \geq 1}$ of the operator $H_{z}$ and the associated eingenvalues $\left(E_{k}\right)_{k>1}$. Remark that Assumption 1.1 implies the operator $H_{z}$ is self-adjoint (see e.g. [RS], vol 2, Theorem X.28) and, defined as a sum of quadratic forms, is an operator with compact resolvent (see [RS], vol. 4, Theorem XIII.67). It possesses purely discrete spectrum and a complete set of eigenfunctions. The reduced model (1.10), (1.11) can be projected on the $\chi_{k}$ 's and is equivalent to the system

$$
\begin{gathered}
i \partial_{t} \varphi_{k}=-\partial_{x}^{2} \varphi_{k}+\frac{1}{2 \pi}\left(\sum_{j=1}^{\infty}\left|\varphi_{j}\right|^{2}\right) \varphi_{k}, \\
\varphi_{k}(0, x)=\int_{\mathbb{R}} \psi_{0}(x, z) \chi_{k}(z) d z, \quad k \in \mathbb{N}^{*} .
\end{gathered}
$$

The solution of the rescaled initial problem is then -formally- asymptotically close to

$$
\psi^{\varepsilon}(t, x, z)=\sum_{k=1}^{\infty} e^{-i t E_{k} / \varepsilon^{2}} \varphi_{k}(t, x) \chi_{k}(z) .
$$

1.3. Statement of the main result. Let us introduce the energy space

$$
\mathcal{H}=\left\{u \in H^{1}\left(\mathbb{R}^{3}\right), \sqrt{V_{c}} u \in L^{2}\left(\mathbb{R}^{3}\right)\right\},
$$

endowed with the norm

$$
\|u\|_{\mathcal{H}}^{2}=\|u\|_{H^{1}\left(\mathbb{R}^{3}\right)}^{2}+\left\|\sqrt{V_{c}} u\right\|_{L^{2}\left(\mathbb{R}^{3}\right)}^{2}=\left\|\partial_{x} u\right\|_{L^{2}\left(\mathbb{R}^{3}\right)}^{2}+\left\|H_{z}^{1 / 2} u\right\|_{L^{2}\left(\mathbb{R}^{3}\right)}^{2} .
$$

Remark that Assumption 1.1 yields the following control for functions in the energy space:

$$
\forall u \in \mathcal{H} \quad \int_{\mathbb{R}^{3}}|z|^{\alpha}|u|^{2} d x d z \leq C\|u\|_{\mathcal{H}}^{2} .
$$

Consider for the rescaled starting model (1.5)-(1.7) a sequence of initial data $\left(\psi_{0}^{\varepsilon}\right)_{\varepsilon>0}$ satisfying the following assumption.

Assumption 1.2. The sequence $\left(\psi_{0}^{\varepsilon}\right)_{\varepsilon>0}$ is uniformly bounded in $\mathcal{H}$ and converges in $L^{2}\left(\mathbb{R}^{3}\right)$ to a function $\psi_{0}$. 
Standard techniques [BM], [Cas], [Caz], [ILZ], allow to prove that for any $\varepsilon \in(0,1)$ the three-dimensional Schrödinger-Poisson system (1.5) - (1.7) admits a unique global weak solution $\left(\psi^{\varepsilon}, V^{\varepsilon}\right)$ for $t \in \mathbb{R}$, in the energy space. In order to analyze its limit as $\varepsilon \rightarrow 0$, let us summarize the available estimates on $\psi^{\varepsilon}$. The first one is the $L^{2}$ estimate. For all $t$ we have

$$
\left\|\psi^{\varepsilon}(t)\right\|_{L^{2}\left(\mathbb{R}^{3}\right)}^{2}=\left\|\psi_{0}^{\varepsilon}\right\|_{L^{2}\left(\mathbb{R}^{3}\right)}^{2} \leq C .
$$

Unfortunately, (see Proposition 2.1), this estimate alone does not enable to bound the selfconsistent potential, and one needs at least an estimate on the derivative of $\psi^{\varepsilon}$ with respect to $x$. Let us now examine the second natural estimate for the Schrödinger-Poisson system, namely the energy estimate. It reads, in rescaled variables:

$$
\begin{aligned}
& \left\|\partial_{x} \psi^{\varepsilon}(t)\right\|_{L^{2}\left(\mathbb{R}^{3}\right)}^{2}+\frac{1}{\varepsilon^{2}}\left\|H_{z}^{1 / 2} \psi^{\varepsilon}(t)\right\|_{L^{2}\left(\mathbb{R}^{3}\right)}^{2}+\frac{1}{|\log \varepsilon|}\left\|V^{\varepsilon}(t)\left|\psi^{\varepsilon}(t)\right|^{2}\right\|_{L^{1}\left(\mathbb{R}^{3}\right)} \\
& =\left\|\partial_{x} \psi_{0}^{\varepsilon}\right\|_{L^{2}\left(\mathbb{R}^{3}\right)}^{2}+\frac{1}{\varepsilon^{2}}\left\|H_{z}^{1 / 2} \psi_{0}^{\varepsilon}\right\|_{L^{2}\left(\mathbb{R}^{3}\right)}^{2}+\frac{1}{|\log \varepsilon|}\left\|V^{\varepsilon}(0)\left|\psi_{0}^{\varepsilon}\right|^{2}\right\|_{L^{1}\left(\mathbb{R}^{3}\right)} .
\end{aligned}
$$

Multiplying this equation by $\varepsilon^{2}$, one can deduce a bound for $\left\|H_{z}^{1 / 2} \psi^{\varepsilon}(t)\right\|_{L^{2}\left(\mathbb{R}^{3}\right)}$ (see the beginning of Section 3), but not for $\left\|\partial_{x} \psi^{\varepsilon}(t)\right\|_{L^{2}\left(\mathbb{R}^{3}\right)}$. However, for a certain set of well-prepared initial data it can be easily proved that this quantity is bounded. As it was remarked in [BMSW], it suffices to consider initial data which are polarized on the first eigenmode $\chi_{1}$ of the transverse Hamiltonian $H_{z}$. This leads to the following theorem, which is our main result.

Theorem 1.3. Under Assumptions 1.1 and 1.2, assume moreover that the initial data is nearly polarized on the first eigenmode $\chi_{1}$ of $H_{z}$, associated to the eigenvalues $E_{1}$ in the sense:

$$
\left\|\left(H_{z}-E_{1}\right)^{1 / 2} \psi_{0}^{\varepsilon}\right\|_{L^{2}\left(\mathbb{R}^{3}\right)} \leq C \varepsilon
$$

Then there exist $C>0$ such that the solution $\psi^{\varepsilon}$ of (1.5)-(1.7) satisfies:

$$
\left\|\partial_{x} \psi^{\varepsilon}(t)\right\|_{L^{2}} \leq C, \quad \text { independently of } \varepsilon>0 \text { and } t \in \mathbb{R},
$$

and the following convergence result holds, for all $T>0$,

$$
\left\|\psi^{\varepsilon}(t, x, z)-e^{-i t E_{1} / \varepsilon^{2}} \varphi(t, x) \chi_{1}(z)\right\|_{L^{2}\left(\mathbb{R}^{3}\right)} \underset{\varepsilon \rightarrow 0}{\longrightarrow} 0 \text { uniformly on }[-T, T],
$$

where $\varphi(t, x)$ solves the cubic defocusing NLS equation

$$
i \partial_{t} \varphi=-\partial_{x}^{2} \varphi+\frac{1}{2 \pi}|\varphi|^{2} \varphi, \quad \varphi(0, x)=\int_{\mathbb{R}} \psi_{0}(x, z) \chi_{1}(z) d z .
$$

Remark that (1.20) is a particular case of the limit model (1.12), (1.13) derived formally in the previous subsection. The keystone of the convergence proof is the $L^{2}$ estimate (1.19) of $\partial_{x} \psi^{\varepsilon}$. In the general case of initial data bounded in $\mathcal{H}$ but not polarized on the first eigenmode, the following partial result can be proved as an easy extension of Theorem 1.3. Under the assumption that (1.19) holds, the function $e^{i t H_{z} / \varepsilon^{2}} \psi^{\varepsilon}$ converges locally uniformly in $L^{2}\left(\mathbb{R}^{3}\right)$ to the solution $\phi$ of

$$
i \partial_{t} \phi=-\partial_{x}^{2} \phi+\frac{1}{2 \pi}\left\langle|\phi|^{2}\right\rangle \phi, \quad \phi(0, x, z)=\psi_{0}(x, z) .
$$

The outline of the paper is the following. In Section 2, we give an asymptotic expansion as $\varepsilon \rightarrow 0$ of the solution of the rescaled Poisson equation (1.6), for wavefunctions $\psi^{\varepsilon}$ in a suitable functional space. Section 3 is devoted to the proof of Theorem 1.3. We use as a first step the energy estimate for well-prepared data in 
order to get an estimate of $\left\|\partial_{x} \psi^{\varepsilon}\right\|_{L^{2}}$ in that case. We conclude the proof using a stability result for the cubic NLS equation.

\section{Approximation of the Poisson kernel}

In this section, we study the convolution with the Poisson kernel when $\varepsilon$ is close to zero. We consider the Poisson potential $V^{\varepsilon}$, after the rescaling $z \mapsto \varepsilon z, x \mapsto x$, and let $\varepsilon \rightarrow 0$ in (1.6). In order to make a precise statement, let us first recall the definition of the finite part of a singular integral. For $u \in C^{0, \eta}(\mathbb{R}) \cap L^{1}(\mathbb{R})$, with $\eta \in(0,1)$, we have

$$
\begin{aligned}
\mathrm{FP} \int_{\mathbb{R}} \frac{u\left(x^{\prime}\right)}{\left|x-x^{\prime}\right|} d x^{\prime} & =\lim _{\eta \rightarrow 0}\left(\int_{\left|x-x^{\prime}\right|>\eta} \frac{u\left(x^{\prime}\right)}{\left|x-x^{\prime}\right|} d x^{\prime}+2 u(x) \log \eta\right) . \\
& =\int_{\left|x-x^{\prime}\right|<1} \frac{u\left(x^{\prime}\right)-u(x)}{\left|x-x^{\prime}\right|} d x^{\prime}+\int_{\left|x-x^{\prime}\right|>1} \frac{u\left(x^{\prime}\right)}{\left|x-x^{\prime}\right|} d x^{\prime} .
\end{aligned}
$$

Both quantities are well defined whenever $u \in \mathcal{C}^{0, \eta}(\mathbb{R}) \cap L^{1}(\mathbb{R})$. Our aim here is to prove the following result.

Proposition 2.1. Consider $\psi$ in the energy space $\mathcal{H}$ defined by (1.14), and let

$$
G^{\varepsilon}(\psi)=\int_{\mathbb{R}} \int_{\mathbb{R}^{2}} \frac{\left|\psi\left(x^{\prime}, z^{\prime}\right)\right|^{2}}{\sqrt{\left(x-x^{\prime}\right)^{2}+\varepsilon^{2}\left|z-z^{\prime}\right|^{2}}} d x^{\prime} d z^{\prime} .
$$

Then we have the following asymptotic expansion:

$$
G^{\varepsilon}(\psi)=-2 \log \varepsilon\left\langle|\psi(x, \cdot)|^{2}\right\rangle+R_{1}(\psi)+R_{2}^{\varepsilon}(\psi),
$$

where

$R_{1}(\psi)=-2 \int_{\mathbb{R}^{2}} \log \left|z-z^{\prime}\right|\left|\psi\left(x, z^{\prime}\right)\right|^{2} d z^{\prime}+2 \log 2\left\langle|\psi(x, \cdot)|^{2}\right\rangle+\mathrm{FP} \int_{\mathbb{R}} \frac{\left\langle\left|\psi\left(x^{\prime}, \cdot\right)\right|^{2}\right\rangle}{\left|x-x^{\prime}\right|} d x^{\prime}$

and for all $u \in \mathcal{H}$ we have

$$
\left\|R_{1}(\psi) u\right\|_{L^{2}} \leq C\|\psi\|_{\mathcal{H}}^{2}\|u\|_{\mathcal{H}}, \quad\left\|R_{2}^{\varepsilon}(\psi) u\right\|_{L^{2}} \leq C_{\beta} \varepsilon^{\beta}\|\psi\|_{\mathcal{H}}^{2}\|u\|_{\mathcal{H}},
$$

for all $\beta<\min (1 / 2, \alpha / 2)$, $\alpha$ being defined according to Assumption 1.1.

Remark that this asymptotic expansion shares several terms (the principal term in $\log \varepsilon$ and the FP term) with the expression that was obtained in [CDR], where a different but connected problem was studied. In this paper, effective Hamiltonian were discussed for modeling a transport on the surface of a cylinder with a small radius, in a linear setting.

Proof. Let us first list some useful available estimates deduced from Sobolev embeddings and from (1.15): for all $u \in \mathcal{H}$, we have

$$
\left\|\partial_{x} u\right\|_{L^{2}\left(\mathbb{R}^{3}\right)}+\|u\|_{L_{x}^{\infty} L_{z}^{2}}+\left\|\partial_{z} u\right\|_{L^{2}\left(\mathbb{R}^{3}\right)}+\left\|\left(1+|z|^{\alpha / 2}\right) u\right\|_{L^{2}\left(\mathbb{R}^{3}\right)} \leq C\|u\|_{\mathcal{H}} .
$$


Let us now decompose

$$
\begin{aligned}
G^{\varepsilon}(\psi) & =\int_{\mathbb{R}^{3}} \frac{\left|\psi\left(x^{\prime}, z^{\prime}\right)\right|^{2}}{\sqrt{\left(x-x^{\prime}\right)^{2}+\varepsilon^{2}\left|z-z^{\prime}\right|^{2}}} d x^{\prime} d z^{\prime} . \\
& =\int_{\mathbb{R}^{2}} \int_{\left|x-x^{\prime}\right|<1} \frac{\left|\psi\left(x^{\prime}, z^{\prime}\right)\right|^{2}-\left|\psi\left(x, z^{\prime}\right)\right|^{2}}{\sqrt{\left(x-x^{\prime}\right)^{2}+\varepsilon^{2}\left|z-z^{\prime}\right|^{2}}} d x^{\prime} d z^{\prime} \\
& +\int_{\mathbb{R}^{2}} \int_{\left|x-x^{\prime}\right|<1} \frac{\left|\psi\left(x, z^{\prime}\right)\right|^{2}}{\sqrt{\left(x-x^{\prime}\right)^{2}+\varepsilon^{2}\left|z-z^{\prime}\right|^{2}}} d x^{\prime} d z^{\prime} \\
& +\int_{\mathbb{R}^{2}} \int_{\left|x-x^{\prime}\right| \geq 1} \frac{\left|\psi\left(x^{\prime}, z^{\prime}\right)\right|^{2}}{\sqrt{\left(x-x^{\prime}\right)^{2}+\varepsilon^{2}\left|z-z^{\prime}\right|^{2}}} d x^{\prime} d z^{\prime} \\
& =I_{1}+I_{2}+I_{3} .
\end{aligned}
$$

We first analyze the term $I_{1}$ by rewriting it as

$$
I_{1}=\int_{\mathbb{R}^{2}} \int_{\left|x-x^{\prime}\right|<1} \frac{\left|\psi\left(x^{\prime}, z^{\prime}\right)\right|^{2}-\left|\psi\left(x, z^{\prime}\right)\right|^{2}}{\left|x-x^{\prime}\right|} d x^{\prime} d z^{\prime}+r_{1}^{\varepsilon},
$$

where $r_{1}^{\varepsilon}$ is to be upper-bounded later. Using

$$
\left|\psi(x, z)-\psi\left(x^{\prime}, z\right)\right| \leq C\left|x-x^{\prime}\right|^{1 / 2}\left(\int_{\mathbb{R}}\left|\partial_{x} \psi(y, z)\right|^{2} d y\right)^{1 / 2},
$$

we deduce that the first term in the right-hand side is well defined and can be bounded thanks to (2.4):

$$
\begin{aligned}
\left|\int_{\mathbb{R}^{2}} \int_{\left|x-x^{\prime}\right|<1} \frac{\left|\psi\left(x^{\prime}, z^{\prime}\right)\right|^{2}-\left|\psi\left(x, z^{\prime}\right)\right|^{2}}{\left|x-x^{\prime}\right|} d x^{\prime} d z^{\prime}\right| & \leq C\left\|\partial_{x} \psi\right\|_{L^{2}}\|\psi\|_{L_{x}^{\infty} L_{z}^{2}} \int_{0}^{1} \frac{1}{\xi^{1 / 2}} d \xi \\
& \leq C\|\psi\|_{\mathcal{H}}^{2}
\end{aligned}
$$

In order to estimate the remainder $r_{1}^{\varepsilon}$, we remark that for all $\gamma \in[0,2]$ there holds:

$$
0 \leq \frac{1}{\left|x-x^{\prime}\right|}-\frac{1}{\sqrt{\left(x-x^{\prime}\right)^{2}+\varepsilon^{2}\left|z-z^{\prime}\right|^{2}}} \leq \frac{\varepsilon^{\gamma}\left|z-z^{\prime}\right|^{\gamma}}{\left|x-x^{\prime}\right|^{1+\gamma}}
$$

Pick $\beta$ such that $0<\beta<\min \left(\frac{1}{2}, \frac{\alpha}{2}\right)$ and take $\gamma=\beta$. One can estimate the remainder as

$$
\left|r_{1}^{\varepsilon}\right| \leq C \varepsilon^{\beta} \int_{\mathbb{R}^{2}} \int_{\left|x-x^{\prime}\right|<1} \frac{\left\|\partial_{x} \psi\left(\cdot, z^{\prime}\right)\right\|_{L_{x}^{2}}}{\left|x-x^{\prime}\right|^{1 / 2+\beta}}\left(|z|^{\beta}+\left|z^{\prime}\right|^{\beta}\right)\left(\left|\psi\left(x^{\prime}, z^{\prime}\right)\right|+\left|\psi\left(x, z^{\prime}\right)\right|\right) d x^{\prime} d z^{\prime},
$$

where we used (2.8) and (2.7). By the Cauchy-Schwarz estimate, for all $u \in \mathcal{H}$ we get

$$
\begin{array}{r}
\left\|r_{1}^{\varepsilon} u\right\|_{L^{2}} \leq C \varepsilon^{\beta}\left\|\partial_{x} \psi\right\|_{L^{2}}\left(\left\||z|^{\beta} \psi\right\|_{L^{2}}\|u\|_{L_{x}^{\infty} L_{z}^{2}}+\|\psi\|_{L_{x}^{\infty} L_{z}^{2}}\left\||z|^{\beta} u\right\|_{L^{2}}\right) \int_{0}^{1} \frac{1}{\xi^{1 / 2+\beta}} d \xi \\
+C \varepsilon^{\beta}\left\|\partial_{x} \psi\right\|_{L^{2}}\|u w\|_{L^{2}}
\end{array}
$$

with

$$
w(x):=\int_{\left|x-x^{\prime}\right|<1} \frac{\left(\int_{\mathbb{R}^{2}}\left|z^{\prime}\right|^{2 \beta}\left|\psi\left(x^{\prime}, z^{\prime}\right)\right|^{2} d z^{\prime}\right)^{1 / 2}}{\left|x-x^{\prime}\right|^{1 / 2+\beta}} d x^{\prime} .
$$

The first line of the right-hand side is bounded thanks to (2.4) and $\beta<\frac{1}{2}$. To bound the last term, we use Hölder and Hardy-Littlewood-Sobolev inequalities:

$$
\|u w\|_{L^{2}} \leq\|w\|_{L^{1 / \beta}}\|u\|_{L_{x}^{2 /(1-2 \beta)} L_{z}^{2}} \leq C\left\||z|^{\beta} \psi\right\|_{L^{2}}\|u\|_{L_{x}^{2 /(1-2 \beta)} L_{z}^{2}} \leq C\|\psi\|_{\mathcal{H}}\|u\|_{\mathcal{H}},
$$


where we used (2.4) and the fact that $\beta<\frac{\alpha}{2}$ and $\frac{2}{1-2 \beta}>2$. Finally, we have

$$
\left\|r_{1}^{\varepsilon} u\right\|_{L^{2}} \leq C \varepsilon^{\beta}\|\psi\|_{\mathcal{H}}^{2}\|u\|_{\mathcal{H}} .
$$

For the term $I_{2}$, a direct computation of the integral with respect to $x^{\prime}$ gives

$$
I_{2}=2(-\log \varepsilon+\log 2)\left\langle|\psi(x, \cdot)|^{2}\right\rangle-2 \int_{\mathbb{R}^{2}} \log \left|z-z^{\prime}\right|\left|\psi\left(x, z^{\prime}\right)\right|^{2} d z^{\prime}+r_{2}^{\varepsilon},
$$

with

$$
r_{2}^{\varepsilon}=2 \int_{\mathbb{R}^{2}}\left|\psi\left(x, z^{\prime}\right)\right|^{2} \log \left(\frac{1+\sqrt{1+\varepsilon^{2}\left|z-z^{\prime}\right|^{2}}}{2}\right) d z^{\prime} .
$$

Let us first estimate the dominant term in $(2.9)$. The term $\left\langle|\psi(x, \cdot)|^{2}\right\rangle$ is clearly bounded in $L^{\infty}$ by (2.4). In order to bound the second term

$$
v=\int_{\mathbb{R}^{2}} \log \left|z-z^{\prime}\right|\left|\psi\left(x, z^{\prime}\right)\right|^{2} d z^{\prime}
$$

we remark that

$$
|\log | z-z^{\prime}|| \leq C\left(\frac{\mathbf{1}_{\left|z-z^{\prime}\right|<1}}{\left|z-z^{\prime}\right|^{1 / 2}}+1+|z|^{\alpha / 2}+\left|z^{\prime}\right|^{\alpha / 2}\right)
$$

and from Hardy-Littlewood-Sobolev and Gagliardo-Nirenberg inequalities, we get, pointwise in $x$,

$$
\int_{\left|z-z^{\prime}\right|<1} \frac{1}{\left|z-z^{\prime}\right|^{1 / 2}}\left|\psi\left(x, z^{\prime}\right)\right|^{2} d z^{\prime} \leq C\|\psi(x, \cdot)\|_{L^{4}}^{2} \leq C\|\psi(x, \cdot)\|_{L^{2}}\left\|\partial_{z} \psi(x, \cdot)\right\|_{L^{2}} .
$$

Hence, for all $u \in \mathcal{H}$,

$$
\begin{aligned}
\|u v\|_{L^{2}} \leq C\|\psi\|_{L_{x}^{\infty} L_{z}^{2}}\left\|\partial_{z} \psi\right\|_{L^{2}} & \|u\|_{L_{x}^{\infty} L_{z}^{2}}+C\left\||z|^{\alpha / 2} \psi\right\|_{L^{2}}^{2}\|u\|_{L_{x}^{\infty} L_{z}^{2}} \\
& +C\|\psi\|_{L_{x}^{\infty} L_{z}^{2}}^{2}\left\|\left(1+|z|^{\alpha / 2}\right) u\right\|_{L^{2}} \leq C\|\psi\|_{\mathcal{H}}^{2}\|u\|_{\mathcal{H}},
\end{aligned}
$$

where we used (2.4). Let us now estimate the remainder $r_{2}^{\varepsilon}$. With the above choice of $\beta \leq \frac{1}{2}<2$, we have

$$
\log \left(\frac{1+\sqrt{1+t^{2}}}{2}\right) \leq C t^{\beta} \quad \text { for all } t>0,
$$

thus, for all $u \in \mathcal{H}$,

$$
\begin{aligned}
\left\|r_{2}^{\varepsilon} u\right\|_{L^{2}} & \leq C \varepsilon^{\beta}\|\psi\|_{L_{x}^{\infty} L_{z}^{2}}\left(\left\||z|^{\beta} \psi\right\|_{L^{2}}\|u\|_{L_{x}^{\infty} L_{z}^{2}}+\|\psi\|_{L_{x}^{\infty} L_{z}^{2}}\left\||z|^{\beta} u\right\|_{L^{2}}\right) \\
& \leq C \varepsilon^{\beta}\|\psi\|_{\mathcal{H}}^{2}\|u\|_{\mathcal{H}},
\end{aligned}
$$

where we used again (2.4) and $\beta<\frac{\alpha}{2}$.

Consider now the term $I_{3}$, that we write

$$
I_{3}=\int_{\left|x-x^{\prime}\right| \geq 1} \frac{\left\langle\left|\psi\left(x^{\prime}, z^{\prime}\right)\right|^{2}\right\rangle}{\left|x-x^{\prime}\right|} d x^{\prime}+r_{3}^{\varepsilon},
$$

with the following immediate bound for the dominant term:

$$
0 \leq \int_{\left|x-x^{\prime}\right| \geq 1} \frac{\left\langle\left|\psi\left(x^{\prime}, z^{\prime}\right)\right|^{2}\right\rangle}{\left|x-x^{\prime}\right|} d x^{\prime} \leq\|\psi\|_{L^{2}}^{2} .
$$


Moreover, from (2.8), the following estimate can be deduced for the remainder:

$$
\begin{aligned}
\left|r_{3}^{\varepsilon}\right| & \leq C \varepsilon^{\beta} \int_{\mathbb{R}^{2}} \int_{\left|x-x^{\prime}\right| \geq 1} \frac{\left|z-z^{\prime}\right|^{\beta}\left|\psi\left(x^{\prime}, z^{\prime}\right)\right|^{2}}{\left|x-x^{\prime}\right|^{1+\beta}} d x^{\prime} d z^{\prime} \\
& \leq C \varepsilon^{\beta}\left(|z|^{\beta}\|\psi\|_{L^{2}}^{2}+\left\||z|^{\beta / 2} \psi\right\|_{L^{2}}^{2}\right) .
\end{aligned}
$$

This is enough to conclude that

$$
\left\|r_{3}^{\varepsilon} u\right\|_{L^{2}} \leq C \varepsilon^{\beta}\|\psi\|_{\mathcal{H}}^{2}\|u\|_{\mathcal{H}}
$$

To complete the proof of the proposition, it suffices to gather (2.6), (2.9) and (2.10), then to use (2.1).

\section{Proof of the main theorem}

As we said in the introduction, the system (1.5)-(1.7) admits two natural conservation laws: the conservation of the $L^{2}$ norm (1.16) and the energy estimate (1.17). Whereas it is immediate to deduce from the first one a uniform estimate of the $L^{2}$ norm of $\psi^{\varepsilon}$, let us examine the second one. Multiplied by $\varepsilon^{2}$, it gives

$$
\left\|H_{z}^{1 / 2} \psi^{\varepsilon}(t)\right\|_{L^{2}\left(\mathbb{R}^{3}\right)}^{2} \leq \varepsilon^{2}\left\|\partial_{x} \psi_{0}^{\varepsilon}\right\|_{L^{2}\left(\mathbb{R}^{3}\right)}^{2}+\left\|\psi_{0}^{\varepsilon}\right\|_{\mathcal{H}}^{2}+\frac{\varepsilon^{2}}{|\log \varepsilon|}\left\|V^{\varepsilon}(0)\left|\psi_{0}^{\varepsilon}\right|^{2}\right\|_{L^{1}\left(\mathbb{R}^{3}\right)} .
$$

From Proposition 2.1 and the Cauchy-Schwarz inequality, we get

$$
\begin{aligned}
\frac{1}{|\log \varepsilon|}\left\|V^{\varepsilon}(0)\left|\psi_{0}^{\varepsilon}\right|^{2}\right\|_{L^{1}} \leq & \frac{1}{|\log \varepsilon|}\left\|V^{\varepsilon}(0) \psi_{0}^{\varepsilon}\right\|_{L^{2}}\left\|\psi_{0}^{\varepsilon}\right\|_{L^{2}} \\
& =\frac{1}{4 \pi|\log \varepsilon|}\left\|G^{\varepsilon}\left(\psi_{0}\right) \psi_{0}^{\varepsilon}\right\|_{L^{2}}\left\|\psi_{0}^{\varepsilon}\right\|_{L^{2}} \leq C\left\|\psi_{0}^{\varepsilon}\right\|_{\mathcal{H}}^{4}
\end{aligned}
$$

thus

$$
\left\|H_{z}^{1 / 2} \psi^{\varepsilon}(t)\right\|_{L^{2}\left(\mathbb{R}^{3}\right)}^{2} \leq\left\|\psi_{0}^{\varepsilon}\right\|_{\mathcal{H}}^{2}+C \varepsilon^{2}\left\|\psi_{0}^{\varepsilon}\right\|_{\mathcal{H}}^{4},
$$

which is uniformly bounded, thanks to Assumption 1.2. In order to have a bound for $\psi^{\varepsilon}$ in the energy space $\mathcal{H}$, it remains to bound the $L^{2}$ norm of $\partial_{x} \psi^{\varepsilon}$. This is done in the next subsection for well-prepared initial data.

3.1. Energy estimate for well-prepared data. We name "well-prepared data" a sequence of initial data $\left(\psi_{0}^{\varepsilon}\right)_{\varepsilon>0}$ in $\mathcal{H}$ which are polarized on the first eigenmode $\chi_{1}$ of $H_{z}$, associated to the eigenvalue $E_{1}$ in the sense (1.18).

We now prove that, under Assumptions 1.1, 1.2 and the assumption of wellprepared data, estimate (1.19) holds true. This only relies on the two conservation laws (1.16) and (1.17). Since $E_{1}$ is the bottom of the spectrum of $H_{z}$, we have

$$
\left\|H_{z}^{1 / 2} u\right\|_{L^{2}}^{2}-E_{1}\|u\|_{L^{2}}^{2}=\int_{\mathbb{R}^{3}} \bar{u}\left(H_{z}-E_{1}\right) u d x d z=\left\|\left(H_{z}-E_{1}\right)^{1 / 2} u\right\|_{L^{2}}^{2},
$$

thus substracting $\frac{E_{1}}{\varepsilon^{2}} \times(1.16)$ to (1.17) leads to the identity:

$$
\begin{aligned}
\left\|\partial_{x} \psi^{\varepsilon}(t)\right\|_{L^{2}}^{2}+ & \frac{1}{\varepsilon^{2}}\left\|\left(H_{z}-E_{1}\right)^{1 / 2} \psi^{\varepsilon}(t)\right\|_{L^{2}}^{2}+\frac{1}{|\log \varepsilon|}\left\|V^{\varepsilon}(t)\left|\psi^{\varepsilon}(t)\right|^{2}\right\|_{L^{1}} \\
& =\left\|\partial_{x} \psi_{0}^{\varepsilon}\right\|_{L^{2}}^{2}+\frac{1}{\varepsilon^{2}}\left\|\left(H_{z}-E_{1}\right)^{1 / 2} \psi_{0}^{\varepsilon}\right\|_{L^{2}}^{2}+\frac{1}{|\log \varepsilon|}\left\|V^{\varepsilon}(0)\left|\psi_{0}^{\varepsilon}\right|^{2}\right\|_{L^{1}} .
\end{aligned}
$$

By Assumption 1.2, (1.18) and (3.2), the right-hand side of this inequality is bounded independently of $\varepsilon$. Hence

$$
\left\|\partial_{x} \psi^{\varepsilon}(t)\right\|_{L^{2}}^{2}+\frac{1}{\varepsilon^{2}}\left\|\left(H_{z}-E_{1}\right)^{1 / 2} \psi^{\varepsilon}(t)\right\|_{L^{2}}^{2} \leq C .
$$


This estimate has two consequences. First, with (3.3) it gives

$$
\left\|\psi^{\varepsilon}(t)\right\|_{\mathcal{H}} \leq C
$$

uniformly with respect to $t$. Second, this estimate shows that $\psi^{\varepsilon}$ remains polarized on the first mode for all time. More precisely, denote

$$
r^{\varepsilon}(t, x, z)=\psi^{\varepsilon}(t, x, z)-\chi_{1}(z) \int \psi^{\varepsilon}\left(t, x, z^{\prime}\right) \chi_{1}\left(z^{\prime}\right) d z^{\prime} .
$$

Remarking that $\left(H_{z}-E_{1}\right)^{1 / 2} \geq\left(E_{2}-E_{1}\right)^{1 / 2}>0$ in the operator sense on $\mathcal{H}$, it can be deduced from (3.4) the following estimate:

$$
\left\|r^{\varepsilon}(t)\right\|_{\mathcal{H}} \leq C \varepsilon
$$

3.2. The convergence theorem. In this section, we prove the convergence result stated in Theorem 1.3. Let

$$
\psi^{\varepsilon}(t, x, z)=e^{-i t E_{1} / \varepsilon^{2}} \varphi_{1}^{\varepsilon}(t, x) \chi_{1}(z)+r^{\varepsilon}(t, x, z) .
$$

Inserting (3.7) in (1.5) and projecting on $\operatorname{Span}\left(\chi_{1}\right)$ leads to the following equation

$$
i \partial_{t} \varphi_{1}^{\varepsilon}=-\partial_{x}^{2} \varphi_{1}^{\varepsilon}+\frac{e^{i t E_{1} / \varepsilon^{2}}}{|\log \varepsilon|} \int_{\mathbb{R}^{2}} V^{\varepsilon}(t, x, z) \psi^{\varepsilon}(t, x, z) \chi_{1}(z) d z .
$$

To deal with the non linear term, we use the decomposition given by Proposition 2.1, with $V^{\varepsilon}=\frac{1}{4 \pi} G^{\varepsilon}\left(\psi^{\varepsilon}\right)$. Remarking that, by orthogonality, we have

$$
\left\langle\left|\psi^{\varepsilon}\right|^{2}\right\rangle=\left|\varphi_{1}^{\varepsilon}\right|^{2}+\left\langle\left|r^{\varepsilon}\right|^{2}\right\rangle
$$

we get from $(2.2)$

$$
\frac{e^{i t E_{1} / \varepsilon^{2}}}{|\log \varepsilon|} \int_{\mathbb{R}^{2}} V^{\varepsilon}(t, x, z) \psi^{\varepsilon}(t, x, z) \chi_{1}(z) d z=\frac{1}{2 \pi}\left|\varphi_{1}^{\varepsilon}\right|^{2} \varphi_{1}^{\varepsilon}+f^{\varepsilon},
$$

with

$$
f^{\varepsilon}=\frac{1}{2 \pi}\left\langle\left|r^{\varepsilon}\right|^{2}\right\rangle \varphi_{1}^{\varepsilon}+\frac{e^{i t E_{1} / \varepsilon^{2}}}{4 \pi|\log \varepsilon|} \int_{\mathbb{R}^{2}}\left(R_{1}\left(\psi^{\varepsilon}\right)+R_{2}^{\varepsilon}\left(\psi^{\varepsilon}\right)\right) \psi^{\varepsilon} \chi_{1} d z .
$$

We clearly have

$$
\left\|f^{\varepsilon}\right\|_{L^{2}(\mathbb{R})} \leq C\left\|\left\langle\left|r^{\varepsilon}\right|^{2}\right\rangle\right\|_{L^{\infty}(\mathbb{R})}\left\|\psi^{\varepsilon}\right\|_{L^{2}}+\frac{C}{|\log \varepsilon|}\left(\left\|R_{1}\left(\psi^{\varepsilon}\right) \psi^{\varepsilon}\right\|_{L^{2}}+\left\|R_{2}^{\varepsilon}\left(\psi^{\varepsilon}\right) \psi^{\varepsilon}\right\|_{L^{2}}\right) .
$$

In order to bound the first term, we notice that by Cauchy-Schwarz

$$
\left|\partial_{x}\left\langle\left|r^{\varepsilon}\right|^{2}\right\rangle\right|^{1 / 2}=\frac{\left|\mathcal{R} e \int \overline{r^{\varepsilon}} \partial_{x} r^{\varepsilon} d z\right|}{\left\langle\left|r^{\varepsilon}\right|^{2}\right\rangle^{1 / 2}} \leq\left\|\partial_{x} r^{\varepsilon}\right\|_{L_{z}^{2}}
$$

thus by the Sobolev embedding $H^{1}(\mathbb{R}) \hookrightarrow L^{\infty}(\mathbb{R})$

$$
\left\|\left\langle\left|r^{\varepsilon}\right|^{2}\right\rangle\right\|_{L^{\infty}(\mathbb{R})} \leq C\left\|\left\langle\left|r^{\varepsilon}\right|^{2}\right\rangle^{1 / 2}\right\|_{H^{1}(\mathbb{R})}^{2} \leq C\left(\left\|r^{\varepsilon}\right\|_{L^{2}\left(\mathbb{R}^{3}\right)}^{2}+\left\|\partial_{x} r^{\varepsilon}\right\|_{L^{2}\left(\mathbb{R}^{3}\right)}^{2}\right) \leq C \varepsilon^{2},
$$

where we used (3.6). Therefore, one deduces directly from (2.3) and (3.5) that

$$
\left\|f^{\varepsilon}\right\|_{L^{2}(\mathbb{R})} \leq \frac{C}{|\log \varepsilon|} .
$$

Now, the conclusion stems from a stability result for the cubic NLS equation in dimension one. Indeed, the functions $\varphi_{1}^{\varepsilon}$ and $\varphi$ solve respectively

$$
i \partial_{t} \varphi_{1}^{\varepsilon}=-\partial_{x}^{2} \varphi_{1}^{\varepsilon}+\frac{1}{2 \pi}\left|\varphi_{1}^{\varepsilon}\right|^{2} \varphi_{1}^{\varepsilon}+f^{\varepsilon}, \quad \varphi_{1}^{\varepsilon}(0, x)=\int_{\mathbb{R}} \psi_{0}^{\varepsilon}(x, z) \chi_{1}(z) d z .
$$


and

$$
i \partial_{t} \varphi=-\partial_{x}^{2} \varphi+\frac{1}{2 \pi}|\varphi|^{2} \varphi, \quad \varphi(0, x)=\int_{\mathbb{R}} \psi_{0}(x, z) \chi_{1}(z) d z .
$$

We remark that both functions are bounded in $H^{1}(\mathbb{R})$, thus in $L^{\infty}(\mathbb{R})$, uniformly in time. For $\varphi_{1}^{\varepsilon}$ this property is a direct consequence of $(3.5)$, as $\left\|\varphi_{1}^{\varepsilon}\right\|_{H^{1}(\mathbb{R})} \leq\left\|\psi^{\varepsilon}\right\|_{\mathcal{H}}$. For $\varphi$, this stems from the energy conservation for the defocusing NLS equation (3.11) and from the fact that, by Assumption 1.2, the initial data $\varphi(0, \cdot)$ belongs to $H^{1}(\mathbb{R})$. Then it is easily seen that for all $t$ we have

$$
\begin{aligned}
& \left\|\varphi_{1}^{\varepsilon}(t)-\varphi(t)\right\|_{L^{2}} \leq\left\|\psi_{0}^{\varepsilon}-\psi_{0}\right\|_{L^{2}}+\int_{0}^{t}\left(\frac{1}{2 \pi}\left\|\left|\varphi_{1}^{\varepsilon}\right|^{2} \varphi_{1}^{\varepsilon}-|\varphi|^{2} \varphi\right\|_{L^{2}}+\left\|f^{\varepsilon}(s)\right\|_{L^{2}}\right) d s \\
& \quad \leq\left\|\psi_{0}^{\varepsilon}-\psi_{0}\right\|_{L^{2}}+C \int_{0}^{t}\left\|\varphi_{1}^{\varepsilon}(s)-\varphi(s)\right\|_{L^{2}} d s+\int_{0}^{t}\left\|f^{\varepsilon}(s)\right\|_{L^{2}} d s,
\end{aligned}
$$

so it follows from (3.9), from Assumption 1.2 and from the Gronwall lemma that for all $T>0$

$$
\left\|\varphi_{1}^{\varepsilon}-\varphi\right\|_{L^{\infty}\left([-T, T], L^{2}(\mathbb{R})\right)} \underset{\varepsilon \rightarrow 0}{\longrightarrow} 0 .
$$

3.3. Towards a more precise approximation. According to (3.9), the convergence rate in Theorem 1.3 is at most $\mathcal{O}\left(\frac{1}{|\log \varepsilon|}\right)$. To go further, Proposition 2.1 suggests the form of the next term in the approximation of the initial model. Taking into account the $R^{1}$ term, one can consider the following system:

$$
i \partial_{t} \widetilde{\varphi}=-\partial_{x}^{2} \widetilde{\varphi}+\frac{1}{2 \pi}|\widetilde{\varphi}|^{2} \widetilde{\varphi}+\frac{1}{4 \pi|\log \varepsilon|}\left(\gamma|\widetilde{\varphi}|^{2}+\mathrm{FP} \int_{\mathbb{R}} \frac{\left|\widetilde{\varphi}\left(x^{\prime}\right)\right|^{2}}{\left|x-x^{\prime}\right|} d x^{\prime}\right) \widetilde{\varphi}
$$

where

$$
\gamma=-\int_{\mathbb{R}^{4}} \log \left(\frac{\left|z-z^{\prime}\right|^{2}}{4}\right)\left|\chi_{1}(z)\right|^{2}\left|\chi_{1}\left(z^{\prime}\right)\right|^{2} d z d z^{\prime}
$$

and

$$
\widetilde{\varphi}(0, x)=\int_{\mathbb{R}^{2}} \psi_{0}^{\varepsilon}(x, z) \chi_{1}(z) d z .
$$

From the approximation result given by Proposition 2.1, one could expect a better convergence rate:

$$
\left\|\psi^{\varepsilon}(t, x, z)-e^{i t H_{z} / \varepsilon^{2}} \widetilde{\varphi}(t, x) \chi_{1}(z)\right\|_{L^{2}} \leq C \varepsilon^{\beta}
$$

with $\beta>0$ as in Proposition 2.1. At the level of this article, this refined convergence result is a conjecture, as well as the existence of the solution $\widetilde{\varphi}$ of (3.12). These questions will be investigated in a future work.

Acknowledgement. We acknowledge support by the ACI Nouvelles Interfaces des Mathématiques no. ACINIM 176-2004 and by the ACI Jeunes chercheurs no. JC1035, funded by the French ministry of research.

\section{References}

[AFS] T. Ando, B. Fowler, F. Stern, Electronic properties of two-dimensional systems, Rev. Mod. Phys., 54 (1982), 437-672.

[Bas] G. Bastard, Wave Mechanics Applied to Semi-conductor Heterostructures, Les Éditions de Physique, EDP Sciences, Les Ulis Cedex, France, 1992.

[BCM] N. Ben Abdallah, F. Castella, F. Méhats, Time averaging for the strongly confined nonlinear Schrödinger equation, using almost periodicity, to appear in J. Diff. Eq. (2008). 
[BAMP] N. Ben Abdallah, F. Méhats, O. Pinaud, Adiabatic approximation of the SchrödingerPoisson system with a partial confinement, SIAM J. Math. Anal, 36 (2005), pp. 986-1013.

[BMSW] N. Ben Abdallah, F. Méhats, C. Schmeiser, R. M. Weishäupl, The nonlinear Schrödinger equation with strong anisotropic harmonic potential, SIAM J. Math. Anal., 37 (2005), no. 1, 189-199.

[BM] F. Brezzi, P. A. Markowich, The three dimensional Wigner-Poisson Problem : existence, uniqueness and approximation, Math. Methods Appl. Sci. 14 (1991), no. 1, 35-61.

[Cas] F. Castella, $L^{2}$ solutions to the Schrödinger-Poisson system: Existence, uniqueness, time behaviour, and smoothing effects, Math. Models Methods Appl. Sci., 7 (1997), pp. 10511083.

[Caz] T. Cazenave, Semilinear Schrodinger Equations, Lecture Notes AMS, (2003).

[CDR] H. Cornean, P. Duclos, B. Ricaud, Effective models for excitons in carbon nanotubes, Ann. Henri Poincaré 8 (2007), no. 1, 135-163.

[FG] D.K. Ferry, S.M. Goodnick, Transport in Nanostructures, Cambridge University Press, Cambridge, UK, 1997.

[ILZ] R. Illner, H. Lange, P. F. Zweifel, Global Existence, Uniqueness and Asymptotic Behaviour of Solutions of the Wigner-Poisson and Schrödinger-Poisson Systems, Math. Methods Appl. Sci. 17 (1994), no. 5, 349-376.

[RS] M. Reed et B. Simon, Methods of Modern Mathematical Physics, vol. 1-4, Academic Press, New York, San Francisco, London, 1972-1979.

[VW] B. Vinter, C. Weisbuch, Quantum Semiconductor Structures: Fundamentals \& Applications, Academic Press, 1991.

Institut de Mathématiques, Université de Toulouse, France

E-mail address: naoufel@math.univ-toulouse.fr

IRMAR, Université Rennes 1, France

E-mail address: francois.castella@univ-rennes1.fr

IRMAR, Université Rennes 1, France

E-mail address: fanny.fendt@univ-rennes1.fr

IRMAR, Université De Rennes 1, France

E-mail address: florian.mehats@univ-rennes1.fr 